\title{
Orthoptic Evaluation and Treatment in Orbital Fractures
}

\author{
Yvette Braaksma-Besselink, CO, MMedSci, Hinke Marijke Jellema, CO, MMedSci, PhD*
}

KEYWORDS

- Orthoptic evaluation $\bullet$ Binocular single vision $\bullet$ Diplopia $\bullet$ Eye motility $\bullet$ Mechanical strabismus $\bullet$ Prisms

KEY POINTS

- Be aware of a combination of mechanical, neurogenic, and sensory causes of diplopia.

- Check for factors influencing the absence of diplopia, such as trauma-related visual problems, abnormal head posture, or preexistent strabismus.

- The severity of the trauma does not always correlate with the impairment of motility or with the degree of diplopia.

- Always prescribe monocular eye movement therapy.

- Treat patients in the first period after trauma with press-on prisms or different types of occlusion, and plan follow-ups as is deemed necessary according to the diagnosis.

\section{Introduction}

Orbital fractures are common in facial trauma and are reported in as many as $40 \%$ of craniofacial trauma. There is no real consensus with regard to standardized examination or protocolled treatment to determine either the necessity or type of treatment. ${ }^{1}$ Surgical indication, meaning possible orbital repair surgery, is predicated on criteria, such as enophthalmos, restriction of extraocular muscles, type and size of the fracture's defect, degree of diplopia, and clinicoradiological findings. ${ }^{1-3}$

\section{Type of diplopia}

Disturbances in ocular motility in orbital fractures may have different causes, but, typically, the diplopia is incomitant in pattern. Incomitant strabismus causing the diplopia is complex; the deviation is different in the various gaze positions. This makes the choices in multifaceted treatment complex. ${ }^{4}$

Medial wall and orbital floor fractures are the most common fractures. Medial wall fractures often give rise to horizontal diplopia. Vertical diplopia is reported more frequently and a common symptom in orbital floor fractures (Case 1) (Fig. 1). In a majority of cases, this leads to a limitation of elevation and in a lesser degree to limitation of depression. ., $^{-6}$

\section{Mechanisms causing diplopia in orbital fractures}

Strabismus resulting in diplopia may occur in $88 \%$ of the patients with orbital fractures. The cause of strabismus may be

\footnotetext{
Funding: None.

Department of Ophthalmology, Amsterdam University Medical Centers, University of Amsterdam, Meibergdreef 9, Amsterdam $1105 \mathrm{AZ}$, the Netherlands

* Corresponding author.

E-mail address: h.m.jellema@amsterdamumc.nl
}

diverse, often involving multiple mechanisms. One of the causes may lie outside of the orbit due to damage to one of the cranial ocular motor nerves or central/peripheral damage due to contrecoup effect of the brain (stem). ${ }^{7}$ Acquired neurogenic palsies frequently are seen in head trauma. Because the fourth nerve is one of the longest of cranial nerves, it is the most vulnerable of the 3 cranial nerves involved in eye movements (third, fourth, and sixth). ${ }^{7}$

Another cause of strabismus may be damage to the ocular muscle itself: swelling, hemorrhage, or even incarceration of the muscle, although actual entrapment of the muscle is uncommon and seen only in posterior floor fractures. Strabismus is not necessarily caused by damage to the ocular muscle itself; it may be due partly to the herniation of orbital fat and connective tissue into the surrounding sinus with subsequent traction on the muscle sheaths.

There also might be a combination of paralytic and mechanical damage causing strabismus ${ }^{8}$ as well as a sensory cause, meaning that a change in visual acuity disrupts binocular functioning ${ }^{9}$ or even decompensation of preexisting strabismus. Be aware of a combination of mechanical, neurogenic, and sensory causes of diplopia.

\section{Role of orthoptic examination}

To assess which of the mentioned mechanisms, discussed previously, is causing diplopia, it is essential to incorporate an orthoptic examination in the initial referral patterns. ${ }^{4}$ Unfortunately, only a few articles regarding orbital fractures include a quantitative (orthoptic) evaluation of ocular motility. ${ }^{4,5,10,11}$ Not all patients present themselves with typical patterns of diplopia $^{12}$ and there is no significant relation between the severity of injury and eventual ocular motility outcome (Case 1). ${ }^{5,6}$ Diplopia not always is the first concern of patients, initially noticed, or observed at all. ${ }^{13}$ Preexisting strabismus and suppression can be reasons for atypical presentation (Case 


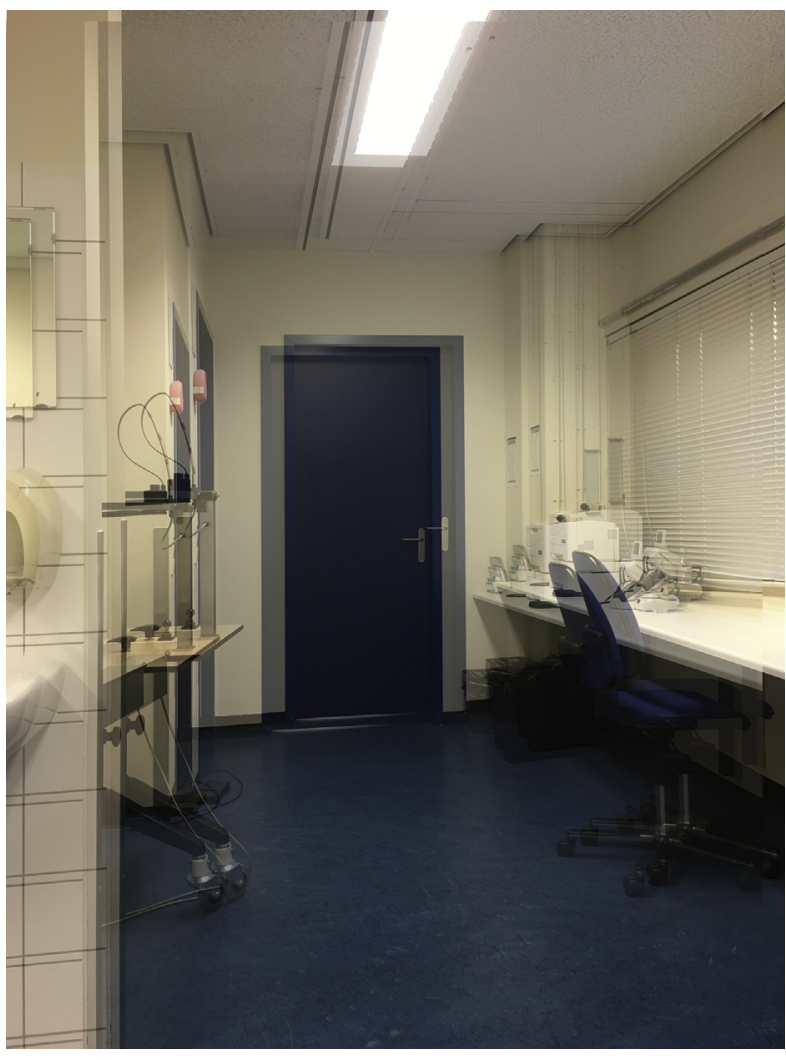

Fig. 1 Vertical diplopia.

2). Suppression is an alteration in visual perception that prevents one of the images to be consciously seen. This results in the absence of diplopia in the presence of strabismus. Other reasons of absence of diplopia are visual field defects, macular problems, vision loss, and neglect. The severity of the trauma does not always correlate with the impairment of motility or with the degree of diplopia.

\section{Timing and frequency of orthoptic examination}

There is overall agreement on the necessity of sequential measurements over the first 4 months to 6 months in the presence of acquired orthoptic abnormalities. ${ }^{13}$ In cases of a neurogenic cause, spontaneous improvement may occur over a time period of 6 months to 9 months. Strabismus secondary to ophthalmologic abnormalities might resolve as vision increases; decompensated strabismus also might resolve spontaneously. Residual diplopia in conservatively treated patients is reported globally, with a significant range, from $15 \%$ to $82 \%$.

Independent of the type of injury, the strabismus is likely to change in the first weeks to months as edema resolves ${ }^{13}$ (Case 3); 7 days to 10 days after trauma, examination is performed more easily because the initial edema often is resolved partially or mostly. Through orthoptic examination, it can be determined if and to what degree diplopia is improved spontaneously and orbital reconstruction may be prevented. ${ }^{6}$ One study of Gosse and colleagues ${ }^{6}$ found no correlation between the type of incomitance and the likelihood of spontaneous recovery; thus, care must be taken in formulating a prognosis in the early stages after trauma.

Strabismus also may occur secondary to orbital reconstruction; an orthoptic examination is advised prior to orbital surgery in cases of suspicion of orthoptic abnormalities to document possible change. ${ }^{14}$ In cases of iatrogenic causes of diplopia after orbital reconstruction, orthoptic evaluation is strongly suggested. ${ }^{14}$ The motility post-orbital surgery can change over a period of time up to 9 months, ${ }^{6}$ so continued follow-up is recommended.

Orthoptic follow-up may lead to different conclusions than primary orbital surgical outcome or may even lead to decisions of further surgery, such as strabismus surgery.

Additionally, it is important to state that after a proper diagnosis, only those with complaints have to be followed-up on.

Orthoptic assessment is important and may play a crucial role in decision making. Each patient must be evaluated individually, however, and the leading practitioner must decide the necessity of orthoptic evaluation based on presentation, severity of trauma, and complaints during the treatment process to avoid excessive examination.

\section{Children}

In cases of an orbital trauma under the age of 8 years, there is a substantial risk for amblyopia. Especially in long-standing strabismus, suppression can arise, which eliminates diplopia but may lead to amblyopia (impaired/delayed visual development, which is permanent if not treated on time). If an orthoptic examination in adults is elective and based on the judgment of the leading physician, an orthoptic examination in a child with orbital fracture(s) is mandatory, at least at some point during the treatment period.

\section{Choice of orthoptic examination}

\section{Taking history, general impression, and ophthalmologic examination}

Patients with orbital trauma often present with periorbital ecchymosis, periocular edema, hemorrhage, enophthalmos, and/or proptosis. When assessing a patient with orbital trauma and possible ocular injury, it is important to determine which ocular structure(s) may be involved. It is imperative to determine whether the injury involves only the orbit or whether the injury may involve the bulb, optic nerve, retina, or other ophthalmic structures. Ocular injury may be indicated by a patient report of monocular diplopia; thus, it is important to distinguish this from binocular diplopia (Box 1). When double vision persists on closing 1 eye, there also is at least monocular diplopia requiring further ophthalmologic examination.

Ocular injury, orbital and retrobulbar hemorrhage, optic nerve damage, retinal injuries, and penetrating eye trauma all constitute ophthalmic emergencies that may threaten vision and require an immediate consult with an ophthalmologist. ${ }^{15}$ Previous studies have found rates of ocular injury in the context of orbital fractures in the range of $26 \%$ to $40 \%$. Ocular trauma is further discussed in detail in Gijsbert J. Hötte and Ronald O.B. de Keizer, article "Ocular injury and emergencies around the globe," in this issue. 


\section{Box 1. Overview of the major causes of monocular and binocular diplopia}

\begin{tabular}{lc}
\hline Monocular Diplopia & Binocular Diplopia \\
\hline - Structural abnormalities & - Disrupted fusion \\
cornea & $\circ$ Head trauma \\
- Structural abnormalities & $\circ$ Visual acuity changes \\
lens & - Strabismus \\
- Structural abnormalities & $\circ$ Preexisting/ \\
retina & decompensation \\
- Refractive error & $\circ$ Acquired \\
& $\circ$ Incomitant eye \\
& movements \\
& $\square$ Mechanical \\
& - Neurogenic \\
\hline
\end{tabular}

It is important to be aware of an abnormal head posture (AHP). The advice is to exclude possible preexisting strabismus, which may be the cause of the AHP. An adopted AHP may mask strabismus and may downplay the extent of complaints. A head posture abnormality may be subtle and easily missed. Be aware of factors influencing the absence of diplopia such as trauma related visual problems, AHP, or preexisting strabismus.

\section{Binocular single vision}

Binocular single vision (BSV) is a unique quality, a combination of 2 images of the same size, color, brightness, and focus to be projected on identical places in both retinae. It is preferable to test the quality of BSV in a natural testing environment, on near or distance fixation. In cases of absent diplopia but present strabismus, regular examinations often are used to determine further treatment cannot be interpreted as normally would be cone. It thus is imperative to determine the extent of binocular function in patients.

\section{Superimposition}

In patients with diplopia, the first impression of the capacity of BSV is to adjust horizontal and/or vertical prisms held in front of the patient's eyes (Fig. 2) in that specific amount in which the patients achieves a single image. This aspect of BSV is called superimposition. Be aware of any possible disruption of superimposition or fusion, such as macular off detachment ${ }^{9}$ or cyclotorsion (tilted image).

\section{Fusion}

When superimposition is reached, fusion measurement gives the investigator information about the quality of BSV. Increasing amounts of prisms (see Fig. 2) are held in front of a patient's eye. The patient's binocular system corrects double vision until a threshold is reached in which the maximum amount of fusion is determined and the patient perceives a double image. The total amount of prisms is called the fusion range. With knowledge about the horizontal and vertical fusional amplitude (range), (Fresnel) prisms can be prescribed to create single vision and to minimize the use of the total

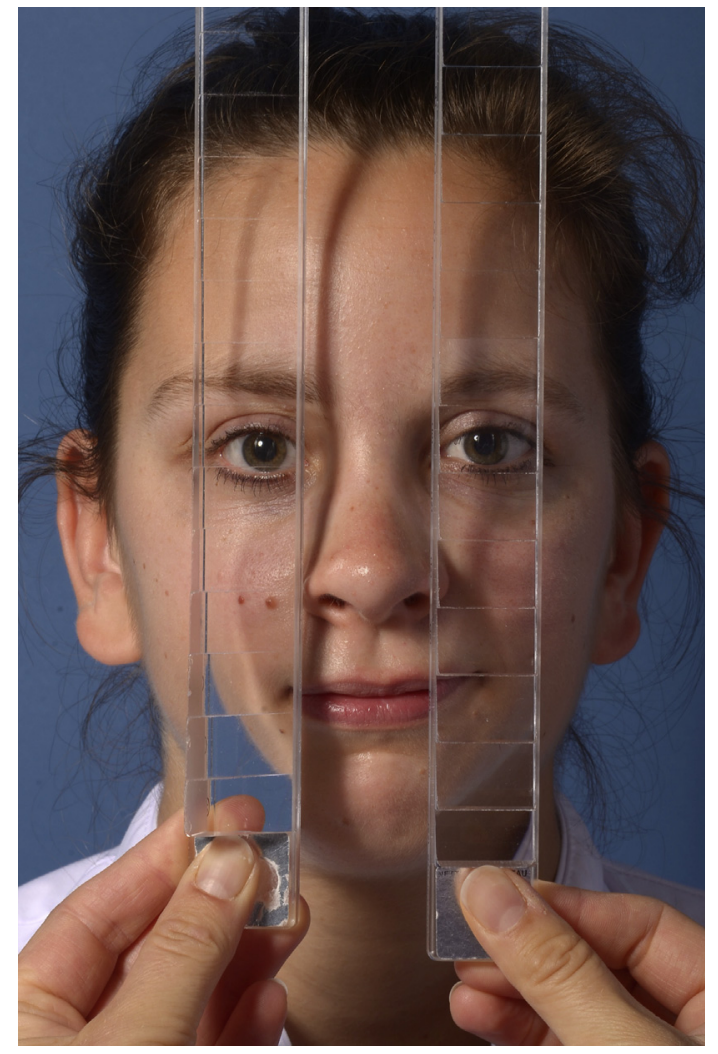

Fig. 2 Horizontal and vertical prisms held in front of the patient's eyes perceiving single vision.

fusion ability and thus minimize complaints. A good capacity of fusion is a positive prognostic factor in patients prior to strabismus surgery.

\section{Field of binocular single vision}

With the field of BSV, the presence or absence of diplopia in all directions of gaze is quantified. For quantifying the field of BSV, different devices are available. In literature, the Goldmann perimeter routinely is used (Fig. 3). This device does not reflect, however, a patient's general viewing condition, because fusion is limited. Measuring the field of BSV (Fig. 4) with the Harmswand or the Maddox screen is performed in more natural viewing conditions.

While measuring the field of BSV, either the patient is asked to follow a light in different directions until double vision is reported or the head is moved while the eyes fixate on a light. Although a field of BSV gives us a way of quantifying double vision, the test outcome may differ significantly from the patient's subjective experience in normal life.

Scoring of the field of BSV can be done with help of the Sullivan core. ${ }^{16}$ With this score, the field of BSV is quantified from 0 (no BSV) to 100 (maximum BSV) (Fig. 5). Quantifying the field of BSV is helpful for research purposes but also helps informing the patients about the progress of the disease.

\section{Eye position and motility}

The amount of strabismus in primary position and other directions of gaze can be measured with different devices. For the primary position, the prism cover test in near and distance 


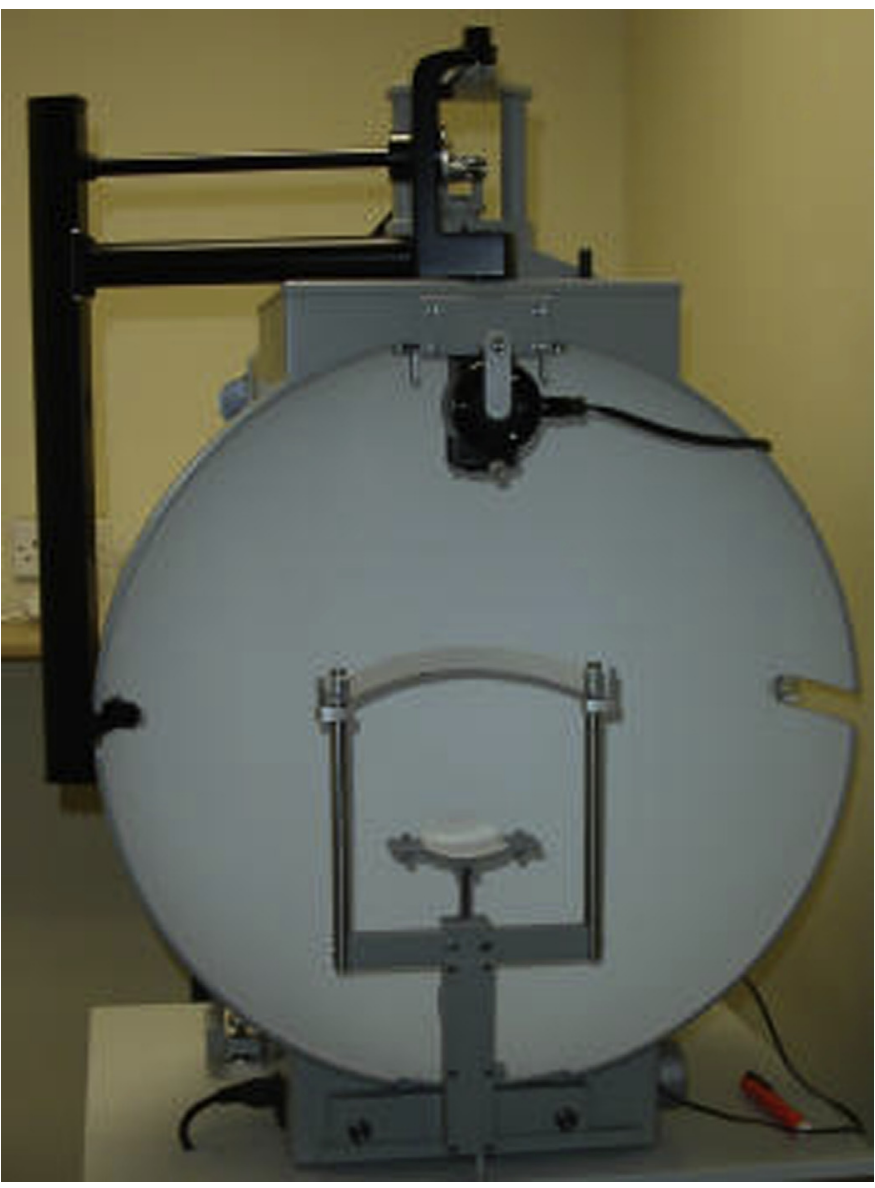

Fig. 3 Goldmann perimeter.

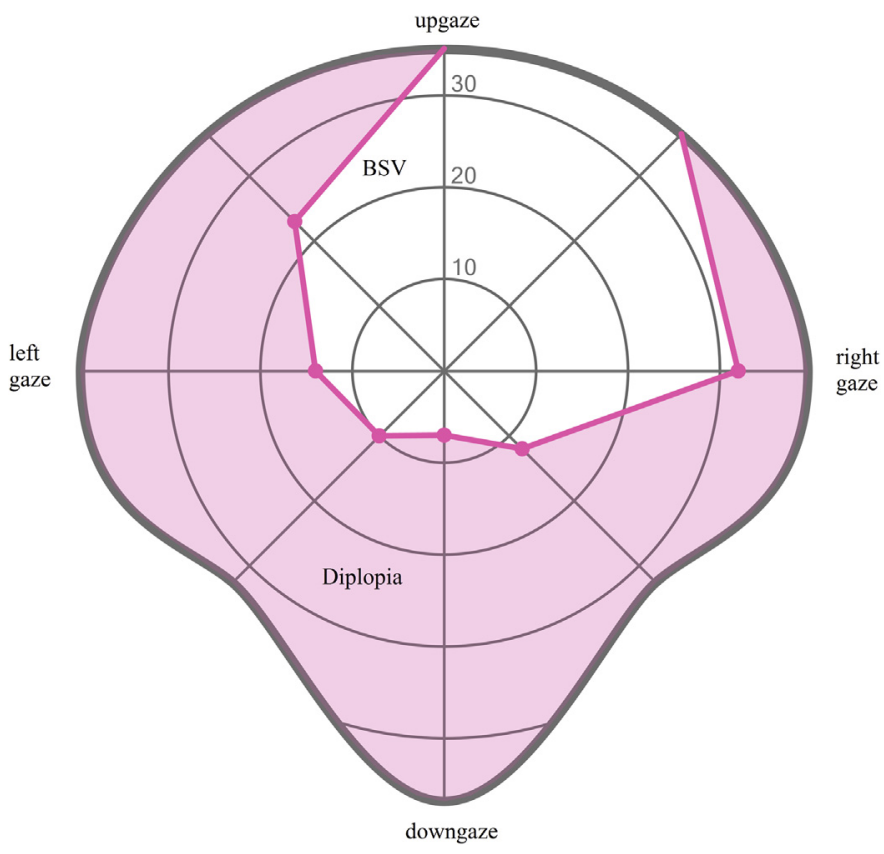

Fig. 4 Example of the field BSV. BSV, binocular single vision.

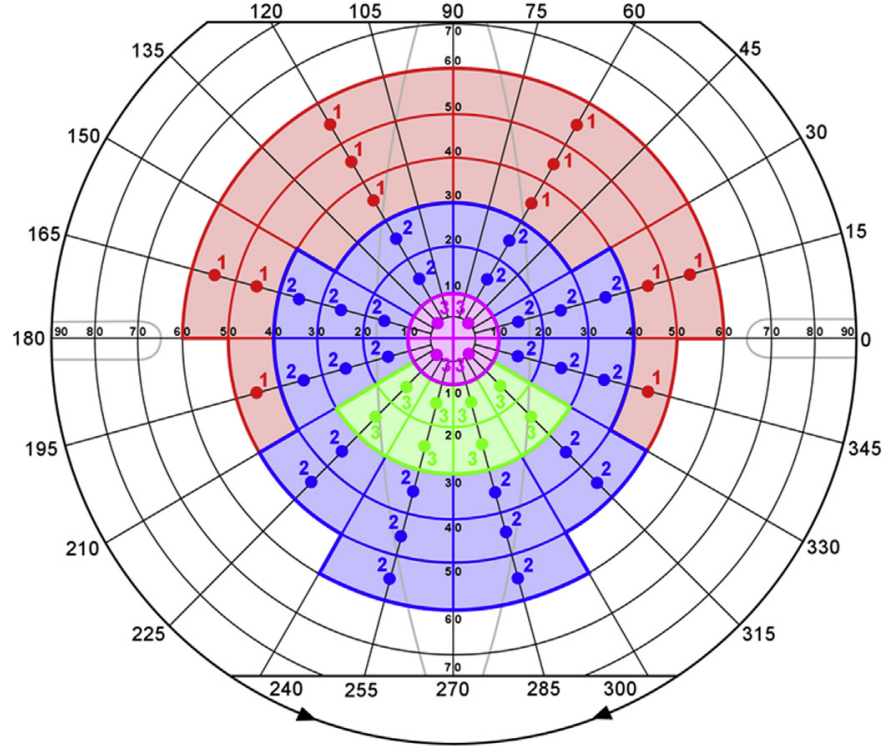

Fig. 5 Field of BSV vision for Goldmann with quantification score of Sullivan. ${ }^{16}$

fixation is used most commonly. A common feature found in patients with traumatic strabismus is the presence of primary and secondary deviation. A secondary deviation means that the amount of strabismus increases when the patient is forced to fixate with an eye that is affected either by mechanical or neurologic restrictions. The eye with the limitation can be identified easily on a motility chart, where the eye movements measured in 9 directions of gaze are visualized. Examples are a Lancaster red-green, a Hess screen, or an Amsterdam motility scheme; the outcome of the diagrams of the aforementioned types of examination, will show a smaller field of measurements for the affected eye (Fig. 6). Alternatively, the cover test in combination with a prism bar can be used in all 9 directions of gaze as well as the Hess-Weis deviometry. ${ }^{4}$

\section{Ductions}

Ductions, meaning monocular eye movement, should be evaluated carefully. Quantifying ductions can be performed in different ways. In general, muscle function is graded on a scale from +4 to -4 . This quantification, however, is observer dependent and less precise compared with a deviometer like the Goldmann ${ }^{17}$ or motility meter (Fig. 7). ${ }^{10,18}$

Ductions generally are measured in 4 positions of gaze: abduction, adduction, elevation, and depression. One article ${ }^{10}$ quantified ductions in the field of action of the 6 extraocular muscles, as suggested by Haggerty and colleagues. ${ }^{19}$

\section{Cyclotorsion}

A majority of patients with diplopia do not experience torsion (tilted image) or do not describe it as such. This is the reason why it is strongly recommended to measure cyclotorsion in all patients with a history of trauma. Measurement of torsion is helpful in identifying oblique muscle weakness and paretic causes, such as a fourth nerve palsy. An excyclotorsion of greater than or equal to $10^{\circ}$ is suspect for a bilateral fourth 

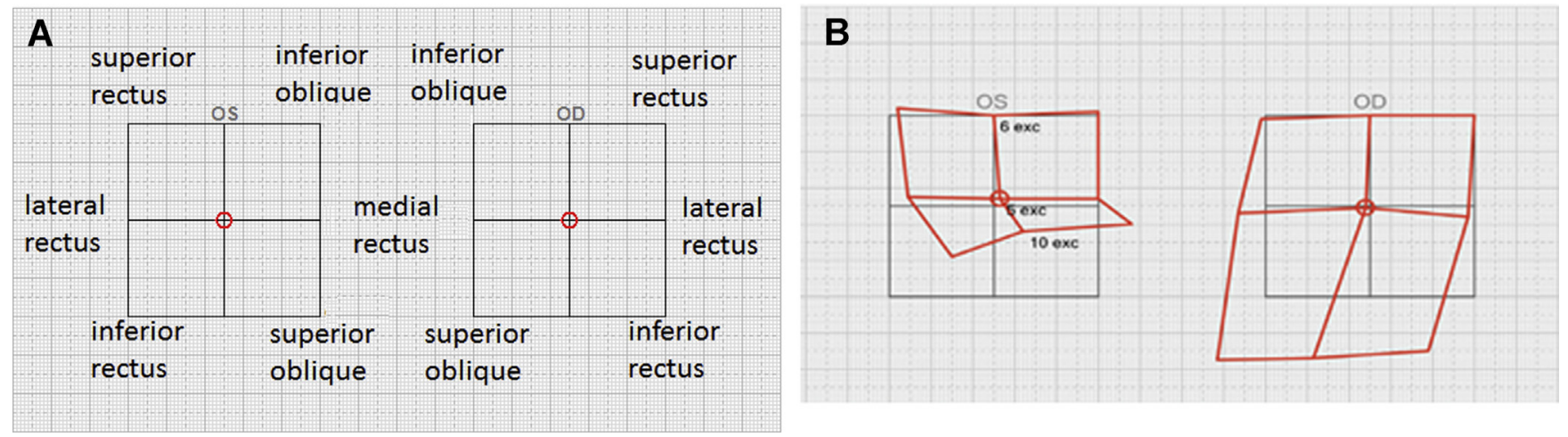

Fig. 6 Amsterdam motility scheme showing the eye movements in nine directions of gaze. (A) Muscle actions in each direction. (B) Patient example with in red the eye position of the patient. Patient with traumatic fourth nerve palsy left $>$ right and an orbital floor fracture of the left side. exc, excyclodeviation; OD, right eye; OS, left eye.

nerve palsy. ${ }^{7}$ Incyclotorsion can be found secondary to orbital injury or repair. ${ }^{20}$

Measurement can be carried out with the Harms tangent screen or Franceschetti cycloforometer of (Fig. 8) in the different directions of gaze. The Maddox double rod test (frame with 2 red glasses) has proved less accurate when measuring more than $10^{\circ}$ to $12^{\circ}$ out of primary position of gaze.

\section{Orthoptic treatment}

In general, all patients are advised to perform monocular eye movement therapy to prevent scarring and maximize ductions.

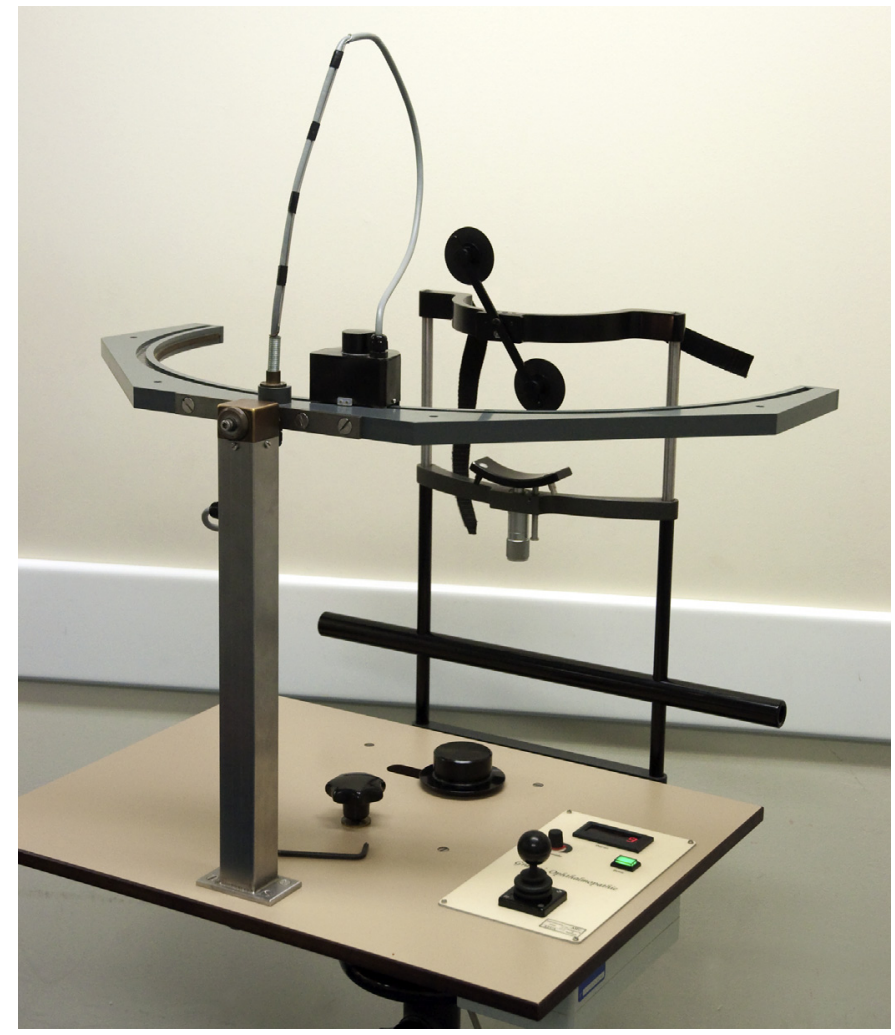

Fig. 7 Motility meter.
There is no literature, however, which subscribes the efficacy of such treatment.

The primary diplopia after trauma and/or new-onset diplopia after repair of the orbital fracture almost always is incomitant and a challenge for treatment. As in all types of treatment, it is important to quantify the primary complaint of the patient and treat accordingly if possible.

The goal of the nonsurgical treatment is to achieve and maintain BSV in primary position and downgaze (reading position), depending on the complaint/profession of the patient. $^{13,21}$ If sufficient BSV is present or the complaints are minor, then conservative treatment is preferred. If not, prisms can be used to regain BSV. Prisms preferably are prescribed as press-on prisms (Fig. 9), because the strabismus may vary over time. If prisms are insufficient, occlusion of 1 eye (Fig. 10) can be advised by means of occlusive foils or an occlusive contact lens. ${ }^{21}$ Treat patients in the first period after trauma with press-on prisms or different types of occlusion.

\section{Strabismus surgery}

Surgical procedures can be performed only if the measurements are unchanging over time. This can take many months

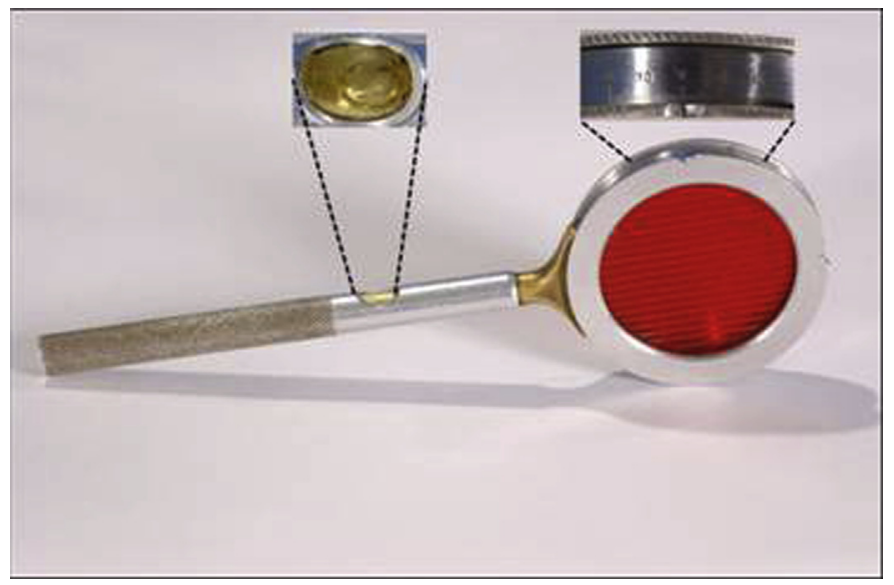

Fig. 8 Cycloforometer of Franceschetti. 

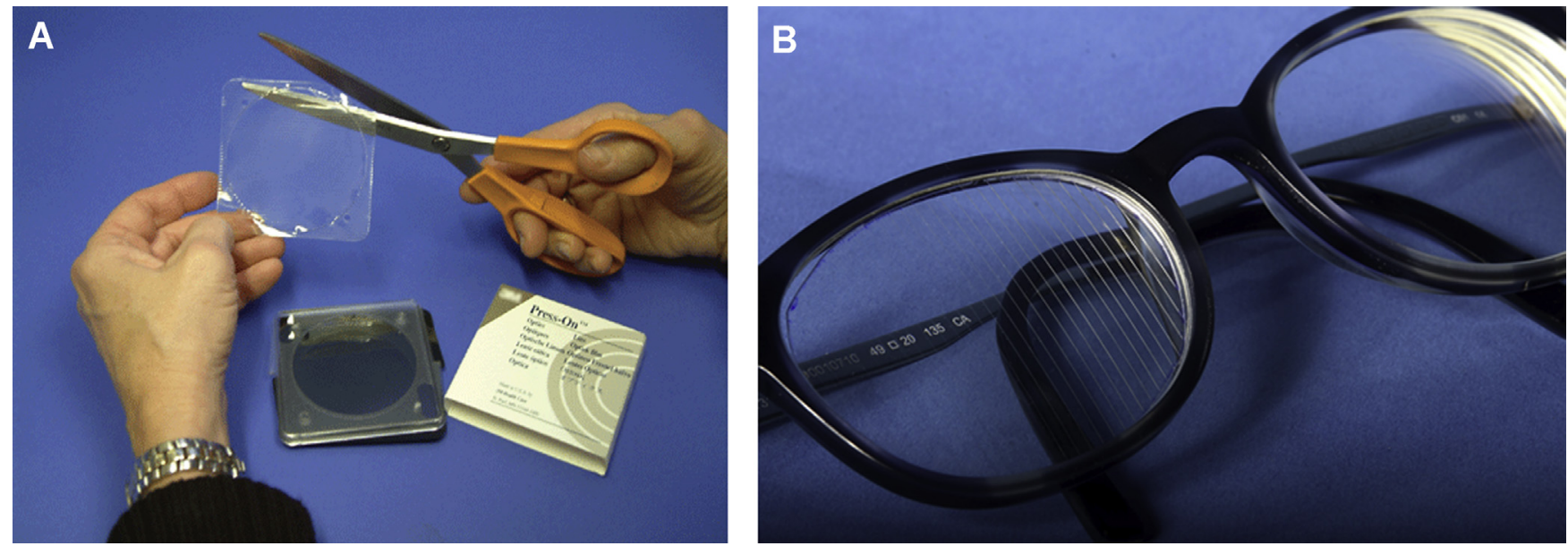

Fig. 9 (A) Press-on prisms. (B) Adjusted to the right lens.

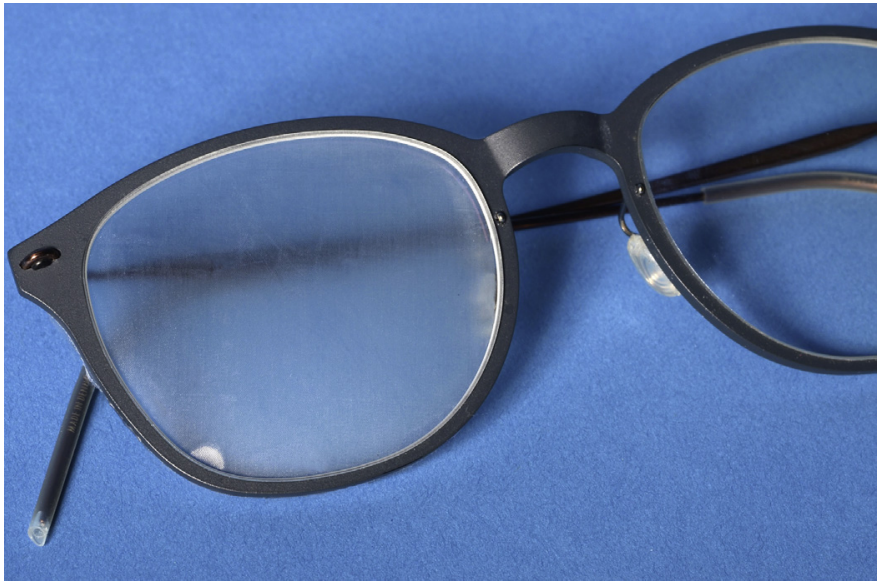

Fig. 10 Occlusion on the right side of the spectacle glass. following the repair of the orbital fracture if the cause is a mechanical one. In general, the time to stability is even longer in cases of a paralytic strabismus. ${ }^{21}$

The primary goal of surgery is to regain BSV in primary and reading gaze or to expand the field of BSV in another field of gaze (Fig. 11).

In a retrospective case series of blowout fractures of Gosse and colleagues, 68 of 80 patients (10\%) eventually needed strabismus surgery, 5 patients of whom achieved comfortable BSV.

Surgically, the treatment depends on whether there is a paretic or mechanical form of strabismus, ${ }^{21}$ but only a few options are possible. Even in cases of strabismus due to orbital surgery, the removal of an orbital implant does not improve the motility. ${ }^{14}$ Generally, the only solution is to place a Faden posterior fixation suture in the overacting eye muscle on the noninjured contralateral eye (Fig. 12). This is in the direction of the damaged muscle to achieve more concomitant eye movements. The goal is to limit the overshooting of the healthy muscle in the other eye, which is an overcompensation of the limited movement on the fractured side. Patients may prefer, however, not to be operated on their sound eye.
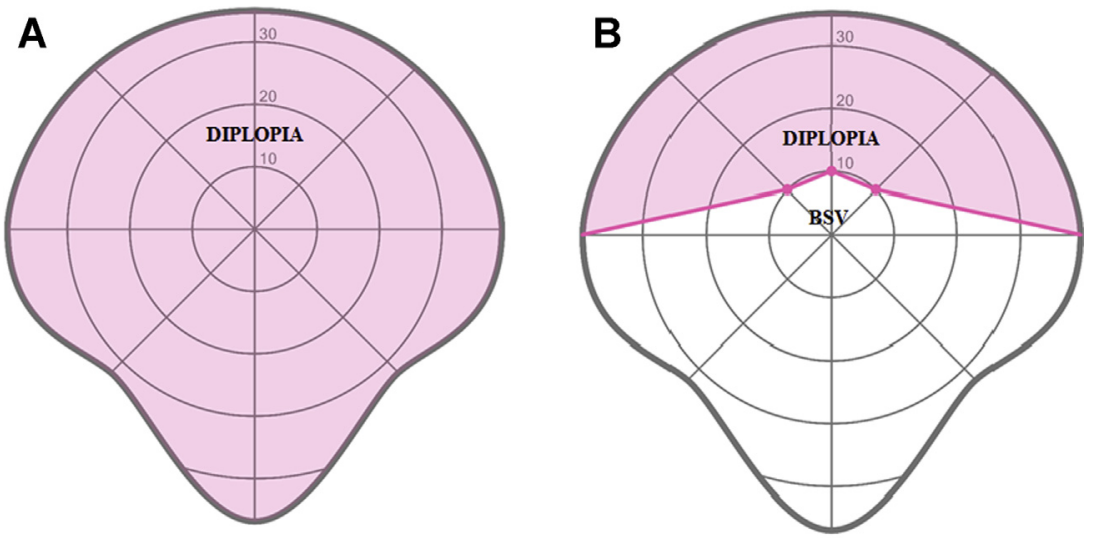

Fig. 11 Field of BSV measured with the Maddox screen. $(A)$ Before surgery and $(B)$ after surgery. BSV, binocular single vision. 

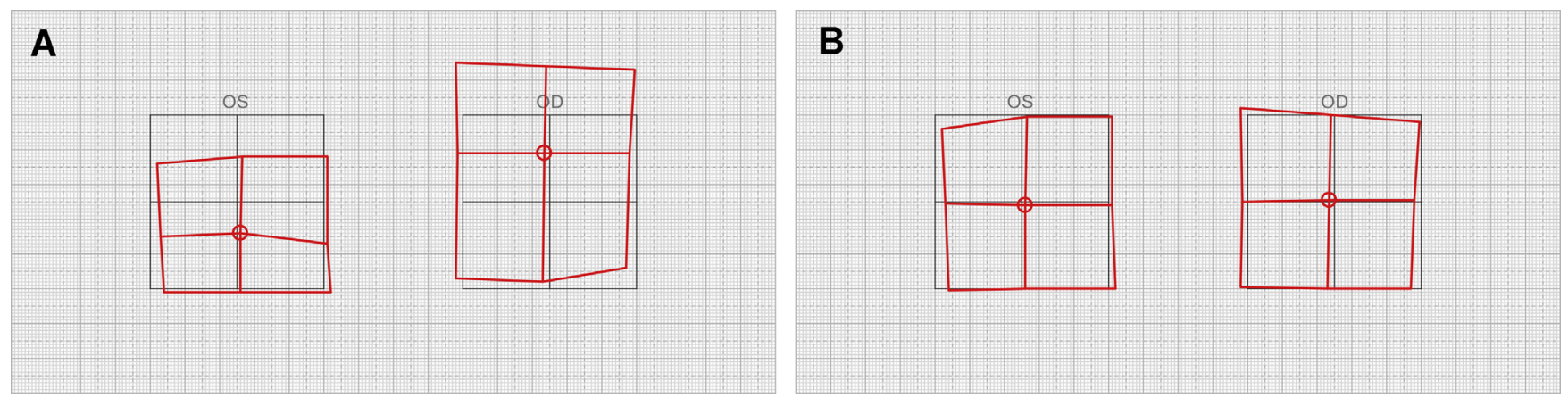

Fig. 12 Eye motility before Faden procedure $(A)$ and after procedure $(B)$. OD, right eye; OS, left eye.

\section{Pearls and pitfalls}

- Check for discrepancies between objective and subjective findings.

- Be aware of other coexisting problems on top of the mechanical causes of the diplopia.

- An orthoptic examination helps in monitoring the recovery process.

- Keep patients comfortable with occlusion and/or prisms until no further change in the orthoptic status are found.

- Diplopia can be treated only by prisms or strabismus surgery.

\section{Conclusion and recommendations}

It is agreed that the orbital fracture is a multifactorial problem that requires a multidisciplinary approach.

Table 1 Details of case 1

\begin{tabular}{lllllll}
\hline & \multicolumn{2}{c}{2 Days } & \multicolumn{2}{c}{10 Days } \\
\hline Eye position & Straight & & & Straight \\
Ductions & & OD & OS & & OD & OS \\
& Elevation & 25 & 29 & Elevation & 24 & 29 \\
& Depression & 48 & 61 & Depression & 60 & 61 \\
\hline Eye motility & Fig. 14 & & Fig. 15 \\
Therapy & Conservative & & Conservative \\
\hline
\end{tabular}

Abbreviation: OD, right eye; OS, left eye.

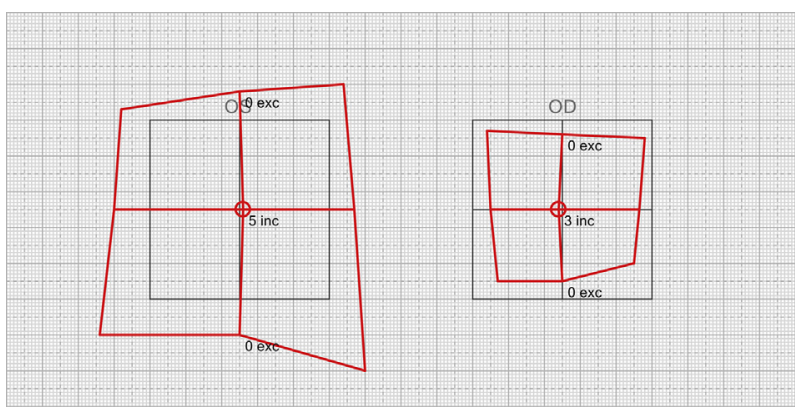

Fig. 14 Eye motility 2 days after trauma (case 1). OD, right eye; OS, left eye.
The orthoptic examination is inextricably associated with patients with orbital fractures. It is too simplistic, however, to state that every patient with orbital trauma or an orbital fracture is in (repeated) need of an orthoptic examination. Each case must be individually deliberated to prevent overtreatment.

\section{Cases}

\section{Case 1}

A 49-year old man with orbital floor fracture due to a soccer accident 2 days ago (Table 1). The CT-scan shows severe herniation of the inferior rectus muscle in the maxillary sinus, including fat (Fig. 13).

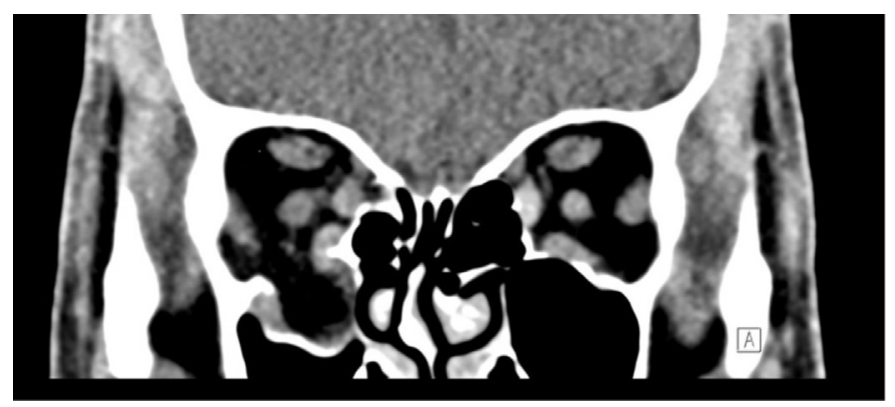

Fig. 13 Orbital floor fracture of the right eye with severe herniation of the inferior rectus in the sinus maxillaris, including fat.

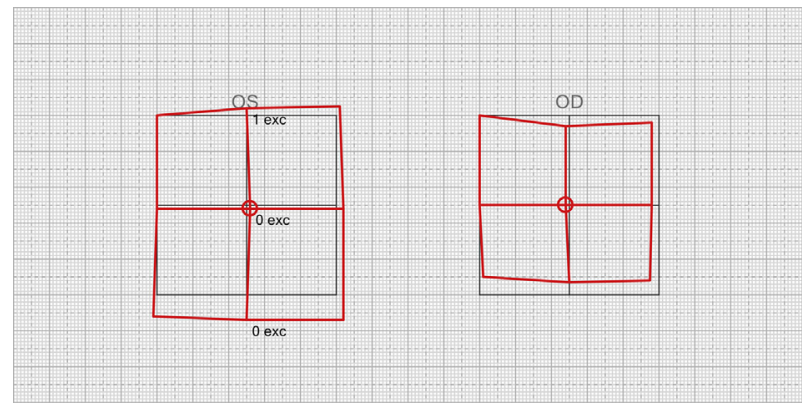

Fig. 15 Eye motility 10 days after trauma (case 1). OD, right eye; OS, left eye. 


\section{Case 2}

A young man presents to the emergency department after highly energetic trauma. He presents with bilateral epidural hematomas with severe facial trauma. There is an orbital floor fracture on both sides as well as infraorbital extraconal hematomas on both sides. (Fig. 16).

Ophthalmic examination 1 day after trauma:

- No ophthalmologic abnormalities, no signs of optic nerve damage

- Patient reports diplopia only on extreme right and left gaze. He is not hindered by this. He experiences tilted image.

- As a child he was treated for strabismus and amblyopia of the right eye

Eye position: small right exotropia and small right hypotropia (no double vision)

BSV and stereopsis: suppression in primary position.

Binocular eye movements:

- Looking to the right: increase of the right hypotropia, maximal in right upgaze

- Looking up: restriction of elevation

Ductions are shown in Table 2.

Conclusion: manifest and incomitant strabismus due to orbital floor fractures and acquired left fourth nerve palsy. Suppression due to preexistent strabismus.

Therapy: wait and reschedule appointment. Recovery of fourth nerve palsy is less than 6 months.

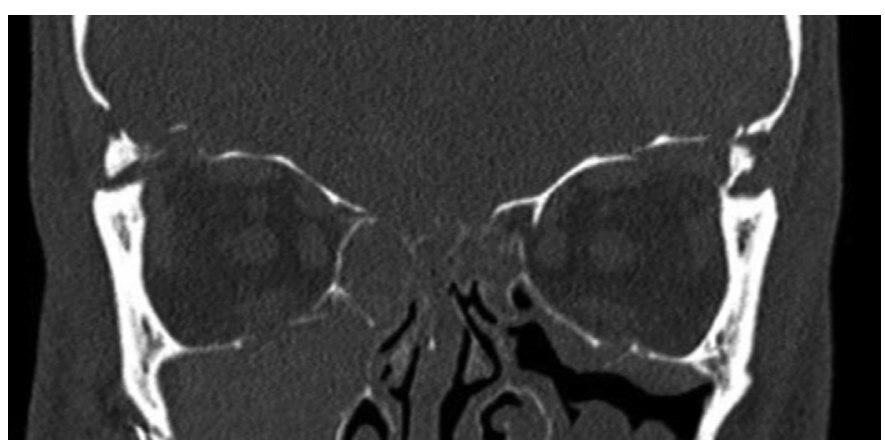

Fig. $16 \mathrm{CT}$ scan of patient showing both orbital floor fractures (case 2).

\section{Case 3}

A 55-year-old man with orbital floor fracture of left orbit and herniation of intraorbital fat in sinus maxillaris (Fig. 17, Table 3).
Table 2 Ductions in case 2

\begin{tabular}{lll}
\hline Duction & OD & OS \\
\hline Abduction & 50 & 44 \\
\hline Adduction & 45 & 45 \\
\hline Elevation & 32 & 54 \\
\hline Depression & 54 & 60 \\
\hline
\end{tabular}

Abbreviation: $\mathrm{OD}$, right eye; OS, left eye.

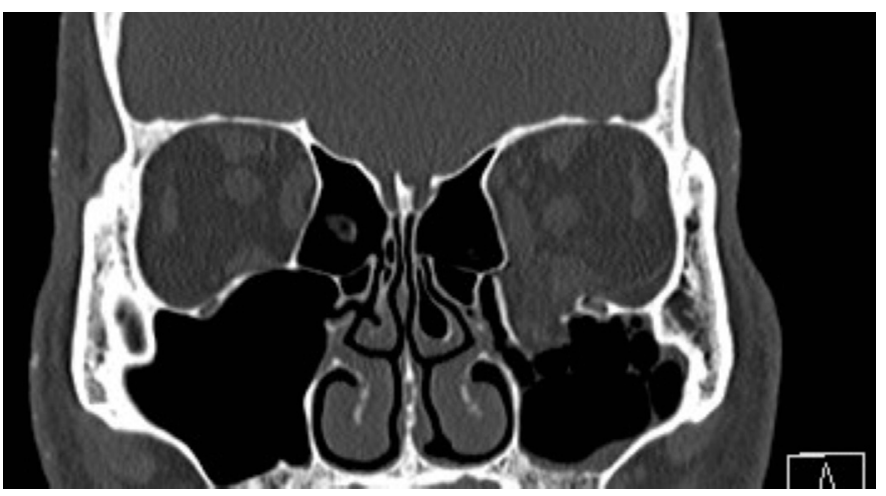

Fig. 17 CT scan showing orbital floor fracture of left orbit (case $3)$.

\section{Disclosure}

The authors have nothing to disclose. 
Table 3 Details of case 3

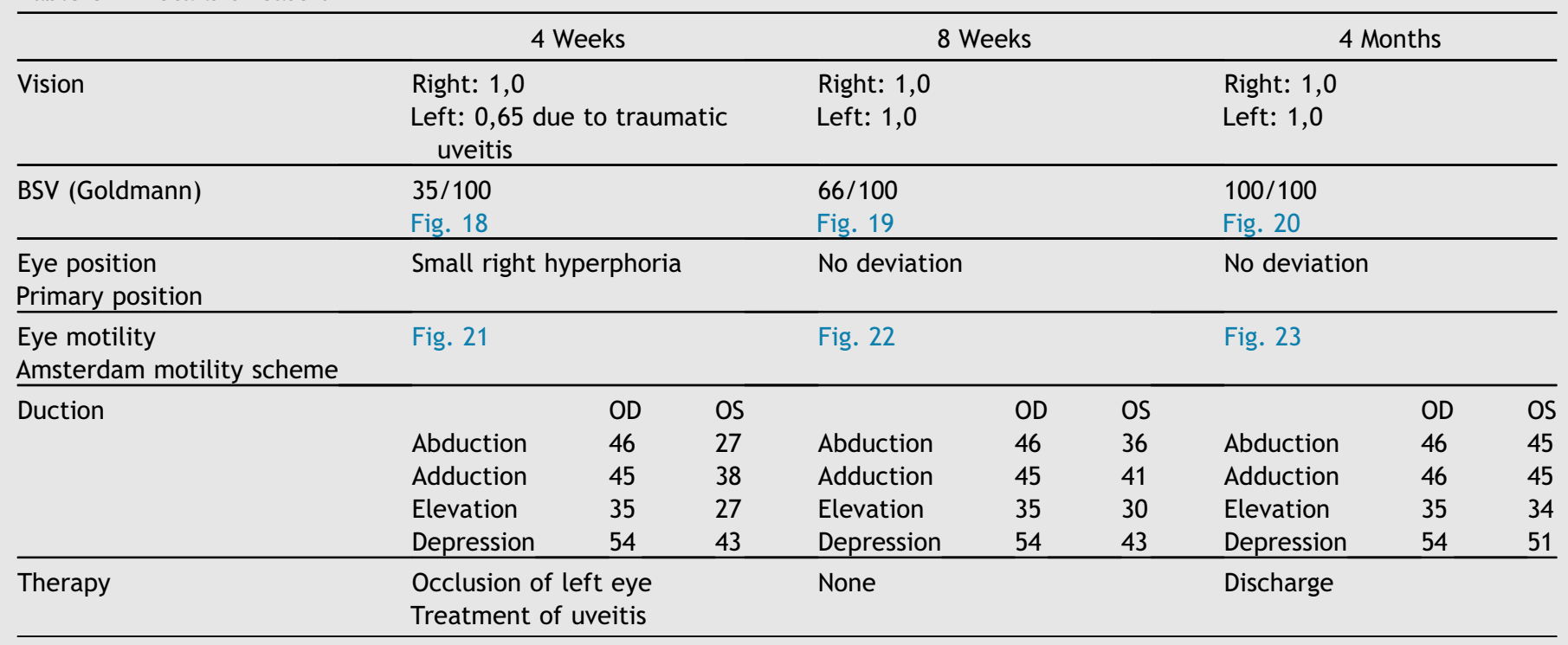

Abbreviation: OD, right eye; OS, left eye.

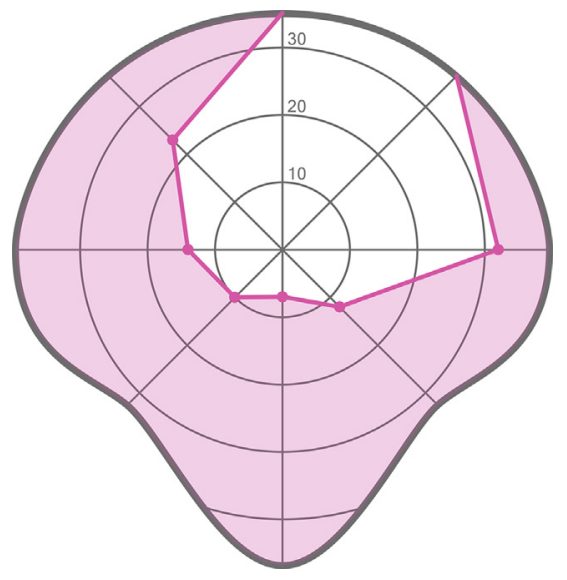

Fig. 18 Field of BSV 4 weeks after trauma (case 3).

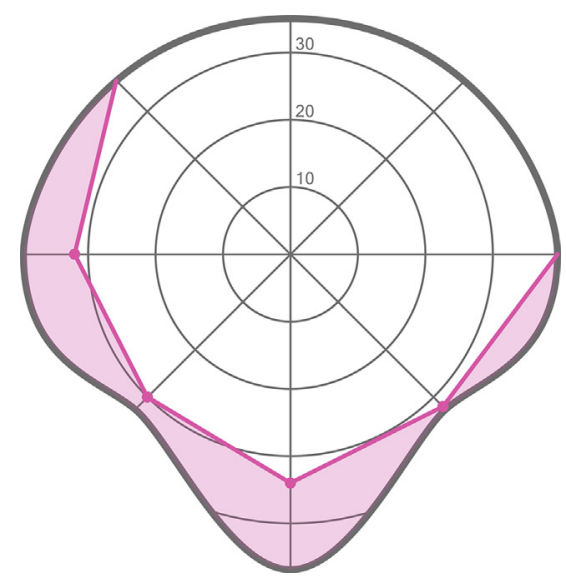

Fig. 19 Field of BSV 8 weeks after trauma (case 3).

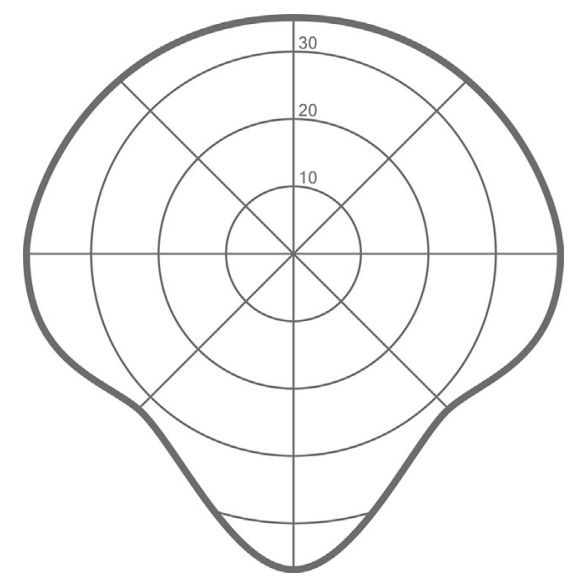

Fig. 20 Field of BSV 4 months after trauma (case 3). 


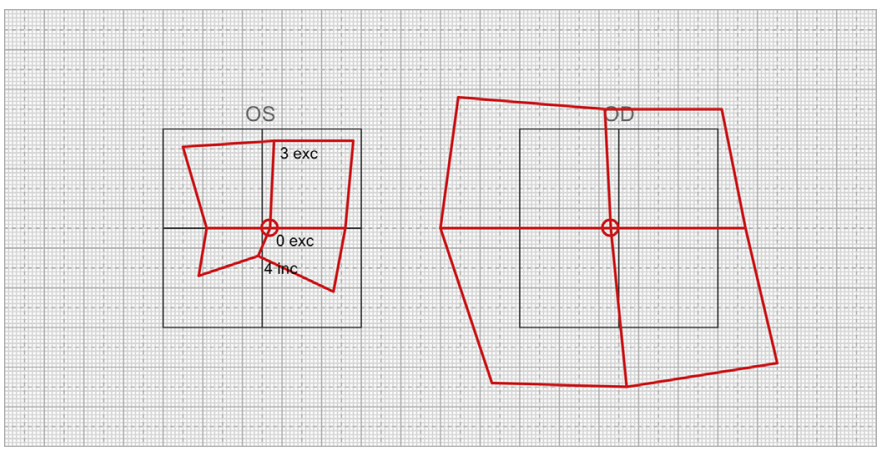

Fig. 21 Eye motility 4 weeks after trauma (case 3). exc, excyclodeviation; OD, right eye; OS, left eye.

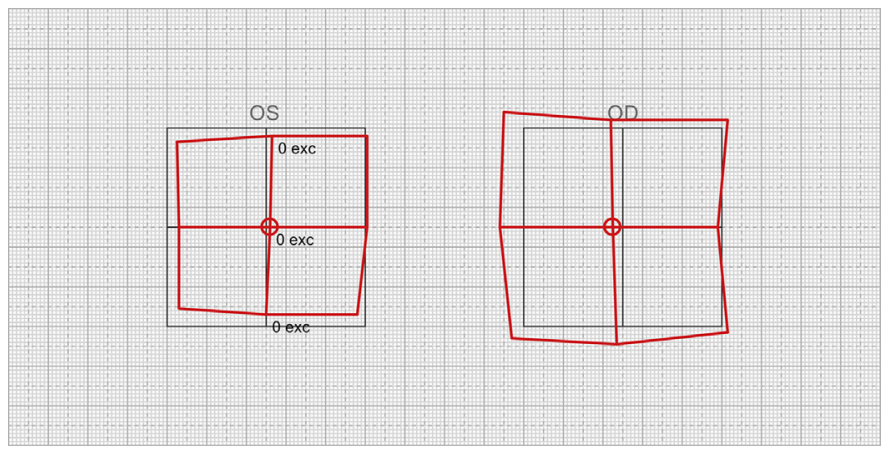

Fig. 22 Eye motility 8 weeks after trauma (case 3). exc, excyclodeviation; OD, right eye; OS, left eye.

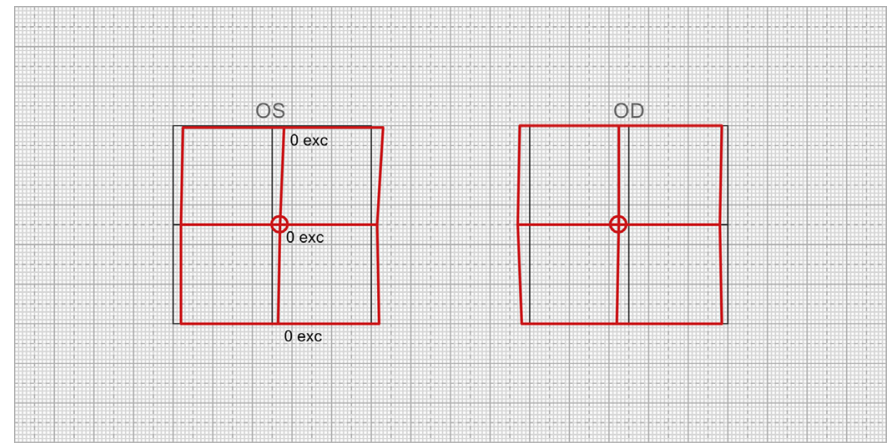

Fig. 23 Eye motility 4 months after trauma (case 3). exc, excyclodeviation; OD, right eye; OS, left eye.

\section{References}

1. Steinegger K, De Haller R, Courvoisier D, et al. Orthoptic sequelae following conservative management of pure blowout orbital fractures: anecdotal or clinically relevant? J Craniofac Surg 2015;26(5):433-7.

2. Marano R, Lino PRS, Zanetti F, et al. Is specialized ophthalmologic evaluation necessary afer orbital fractures? A prospective 64-case study. Oral Maxillofac Surg 2019;23:325-9.

3. Dubois L, Jansen J, Schreurs R, et al. How reliable is the visual appraisal of a surgeon for diagnosing orbital fractures? J Craniomaxillofac Surg 2016;44:1015-24.

4. Bianchi F, De Haller R, Steffen H, et al. Does vertical incomitance predict the diplopia outcome in orbital fracture patients? A prospective study of 188 patients. J Craniomaxillofac Surg 2019;47:305-10.

5. Rowe F, Crowley T. Outcome of ocular motility disturbances in orbital injuries. Strabismus 2003;11(3):179-88.

6. Gosse EM, Ferguson AW, Gilmour C, et al. Blow-out fractures: patterns of ocular motility and effect of surigcal repair. $\mathrm{Br} \mathrm{J}$ Oral Maxillofac Surg 2010;48:40-3.

7. Walonker AF. Diagnostic evaluation of traumatic cranial nerve palsies. Am Orthopt J 2004;54:57-61.

8. Xia Q, Wang Z, Yan J. Surgical management of strabismus in patients with orbital fracture. J Craniofac Surg 2018;29:1865-9.

9. Pritchard C, Ellis GS. Management of central sensory deficits following trauma. Am Orthopt J 2004;54:45-8.

10. Alhamdani F, Durham J, Greenwood M, et al. Diplopia and ocular motility in orbital blow-out fractures: 10-year retrospective study. J Craniomaxillofac Surg 2015;43(7):1010-6.
11. Jansen J, Dubois L, Maal TJJ, et al. A non-surgical approach with repeated orthoptic evaluation is justified for most blow-out fractures. J Craniomaxillofac Surg 2020;48:560-8.

12. Folkestad L, Aberg-Bengtsson L, Granstrom G. Recovery from orbital floor fractures: a prospective study of patients and doctors' experiences. Int J Oral Maxillofac Surg 2006;35:499-505.

13. Arnoldi K, Mattheu J. Diagnosis and nonsurgical management of strabimus secondary to orbital fracture. Am Orthopt J 2004;54: 7-12.

14. Silbert DI, Matta NS, Singman EL. Diplopia secondary to orbital surgery. Am Orthopt J 2012;62:22-8.

15. Ramponi DR, Astorino T, Bessetti-Barrett CR. Orbital floor fractures. Adv Emerg Nurs J 2017;39(4):240-7.

16. Sullivan TJ, Kraft SP, Burack C, et al. A functional scoring method for the field of bincular single vision. Ophthalmology 1992;99:575-81.

17. Gerling J, Lieb B, Kommerell G. Duction ranges in normal probands and patients with Graves' ophthalmopathy, determined using the Goldmann perimeter. Int Ophthalmol 1997;21(4):213-21.

18. Mourits MP, Prummel MF, Wiersinga WM, et al. Measuring eye movements in Graves' ophthalmopathy. Ophthalmology 1994; 101(8):1341-6.

19. Haggerty H, Richardson S, Mitchell KW, et al. A modified method for measuring uniocular fields of fixation: reliability in healthy subjects and in patients with Graves' Orbitopathy. Arch Ophthalmol 2005;123(3):356-62.

20. Roper-Hall G. Diagnosis and management of cetnral motro deficits following trauma. Am Orthopt J 2004;54:49-54.

21. Kerr NC. Management of strabismus following orbital fracture repair. Am Orthopt J 2004;54:24-31. 